Research Paper

\title{
Genetic Differentiation and Efficient Sex-specific Marker Development of a Pair of $Y$ - and $X$-linked Markers in Yellow Catfish
}

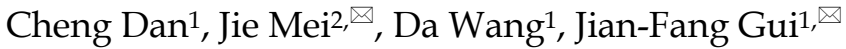 \\ 1. State Key Laboratory of Freshwater Ecology and Biotechnology, Institute of Hydrobiology, Chinese Academy of Sciences, Graduate \\ University of the Chinese Academy of Sciences, Wuhan 430072, China. \\ 2. College of Fisheries, Key Laboratory of Freshwater Animal Breeding, Ministry of Agriculture, Freshwater Aquaculture Collaborative \\ Innovation Center of Hubei Province, Huazhong Agricultural University, Wuhan, 430070, China.
}

$\triangle$ Corresponding author: Tel: +86 27 68780707; E-mail: jfgui@ihb.ac.cn (J.-F. Gui) and jmei@mail.hzau.edu.cn (J. Mei).

(๑) Ivyspring International Publisher. This is an open-access article distributed under the terms of the Creative Commons License (http://creativecommons.org/ licenses/by-nc-nd/3.0/). Reproduction is permitted for personal, noncommercial use, provided that the article is in whole, unmodified, and properly cited.

Received: 2013.07.19; Accepted: 2013.09.18; Published: 2013.1I.05

\begin{abstract}
$P f 62-Y$ and $P f 62-X$ is a pair of allelic $Y$ chromosome-linked and $X$ chromosome-linked markers, and have been used to identify $Y Y$ super-males, $X Y$ males and $X X$ females for commercial production of all-male populations in yellow catfish (Pelteobagrus fulvidraco). However, the SCAR primers used previously have only two nucleotide difference, which restricts the wide utility because of nucleotide polymorphism. In this study, a continuous 8102 bp Pf62-Y sequence and a 5362 bp Pf62-X sequence have been cloned by genome walking, and significant genetic differentiation has been revealed between the corresponding $X$ and $Y$ chromosome allele sequences. Moreover, three pairs of primers were designed to efficiently identify $Y Y$ super-males, $X Y$ males and $X X$ females in an artificial breeding population, and to distinguish $X Y$ males and $X X$ females in various wild populations. Together, the three new sex-specific genetic markers develop a highly stable and efficient method for genetic sex identification and sex control application in sustainable aquaculture of all-male yellow catfish.
\end{abstract}

Key words: Yellow catfish; Sex-specific marker; genome walking; YY super-males; All-male fish.

\section{Introduction}

Sex-specific or sex chromosome-linked markers have significant implications for genetic improvement of economically important traits in some aquaculture fishes [1][2] because of the uncommon growth difference between females and males [3][4][5]. Previously, male- or female-specific DNA markers were isolated from several important aquaculture fishes, such as African catfish Clarias gariepinus [6], Asian arowana (Scleropages formosus) [7], Nile tilapia Oreochromis niloticus [8][9][10], salt tolerant tilapia hybrid (Oreochromis mossambicus x Oreochromis spp.)[11], rainbow trout Oncorhynchus mykiss [12], half-smooth tongue sole Cynoglossus semilaevis [13][14][15], yellow catfish Pelteobagrus fulvidraco [16], common carp Cy- prinus carpio [17][18], Paramisgurnus dabryanus [19], matrinchã Brycon amazonicus [20], Scophthalmus maximus [21], blunt snout bream Megalobrama amblycephala [22], and rock bream Oplegnathus fasciatus [23]. In yellow catfish (Pelteobagrus fulvidraco), Wang et al. [16] identified two pairs of $Y$ chromosome- and X chromosome-linked SCAR markers by AFLP screening from an artificial breeding population[24], and thereby developed a Y- and X-specific allele marker-assisted sex control technique for mass production of all-male population. Because adult yellow catfish males are about two to three fold bigger than the females, the all-male population, as a novel variety "yellow catfish all-male No. 1", has been widely used 
for commercial production throughout China since 2010 [1][25].

Significant implications and application values of the all-male yellow catfish production have been evaluated [1][25][26]. However, these sex-linked SCAR markers used in all-male production were only tested in one artificial breeding population as reported, and the primers of $P f 62-X$ and $P f 62-Y$ have only two nucleotide difference [16]. In order to search for more accurate molecular markers for large-scale all-male production of yellow catfish, we performed genome walking and sequencing of the $Y$ chromosome-linked marker Pf62- $Y$ and $X$ chromosome-linked marker Pf62- $X$, and analyzed the nucleotide divergence and genetic differentiation. Based on these studies, we developed three pairs of new markers that can efficiently identify the genetic sex of yellow catfish in breeding and wild populations.

\section{Materials and Methods}

\section{Source of experimental samples}

The artificial breeding yellow catfish including $X X$ females, $X Y$ males and $Y Y$ super-males came from Institute of Hydrobiology, Chinese Academy of Sciences and from Hubei Daming Aquatic Science-Technology Company (Jingzhou). The wild populations were collected from Liangzi Lake (Wuhan), Chang Lake (Jingzhou), Hong Lake (Honghu), South Lake (Zhongxiang) and Dongting Lake (Hunan).

\section{Genomic DNA extraction}

The genomic DNA was extracted from fin clips of the $X X$ female, $X Y$ male and $Y Y$ super-male individuals using a standard phenol-chloroform method [27], and the quality and concentration of DNA were assessed by ethidium bromide-stained $0.7 \%$ agarose gel under ultraviolet light and by spectrophotometer as described previously [28].

\section{Genome walking}

BD Genome Walker ${ }^{\mathrm{TM}}$ Universal Kit (Clontech) was used to construct the DNA fragment pools. High quality genomic DNA sample extracted from $X X$ female and $Y Y$ super-male was divided into four portions, and four kinds of restriction enzymes: Dra I, EcoR V, Pvu II, Stu I were used to digest the DNA samples for $20 \mathrm{~h}$ respectively. Phenol/chloroform and $80 \%$ ethanol were used to precipitate and rinse the samples, and the digested genomic DNA was dissolved in 1xTE buffer (10 mM Tris, $0.1 \mathrm{mM}$ EDTA). Each batch of digested genomic DNA was then ligated separately to the adapter sequence and used as templates. The adapter primers AP1, AP2 and walking primers listed in Table 1 were designed and synthesized. The genomic walking PCR programs were referred to the manual proposal as described previously [29][30].

The amplified products were examined by $1.0 \%$ agarose gel electrophoresis. Single and clear major bands (>500bp long bands) were extracted and purified from gel, and directly sequenced in both directions [31][32]. Sequence fragments were assembled by DNAMAN software.

To confirm the assembled sequence, a pair of primers, 62F (5'-GATCTGGTGACCTACAAAATTGA CA-3') and 62R (5'-AAGCACGACTGGGCCAATGTT TCCATC-3') was designed according to both ends of the entire sequence. L-A Taq polymerase (Cat.No.A0901A, Takara) was used for amplification reaction through the following PCR program: denaturation in $94^{\circ} \mathrm{C}$ for 30 seconds, annealing and extension at $68^{\circ} \mathrm{C}$ for 9 minutes, 36 cycles. The PCR products were detected and purified by $0.8 \%$ agarose gel electrophoresis, and sequenced as described previously [33].

Table I. Genomic walking primers for cloning Pf62 markers in yellow catfish

left fragment primers $\left(5^{\prime}-3^{\prime}\right)$

GSP-1a: CTCTGTGCGACACGGGGAACGGTGAAACCT

GSP-1b: CCGAGAGACATCTTACCCCAGGGCTTATGG

GSP-2a: TCAAGATGTAAAGATGCCTCCCTTCTCGCT

GSP-2b: GCTTTGATCAGGTTGCTGGATTGCGATGT
Right fragment primers $\left(5^{\prime}-3^{\prime}\right)$

GSPr-1a: CATAAGCCCTGGGGTAAGATGTCTCTCGGA

GSPr-1b: AGGTTTCACCGTTCCCCGTGTCGCACAGA

GSPr-2a: TCTCCCTCAAGCTGTTGTCCTTCAAGATG

GSPr-2b: AACGTGTCGGTGATTTGCATGCGCTCTC

GSPr-3a: GAGCTGTATGCTGGTCTAATAAACACAT

GSPr-3b: CTCCTTGCTCAGTGGCGAAGACATCA

GSPr-4a: CCCTTTTTTCCTTGGGGCTCAAATGAGTC

GSPr-4b: CCTTACAAATGAAGGTTGATGTTGAAGTTG

GSPr-5a: AGATGTAAAGGAGGCAGGTTAGCTGCTG

GSPr-5b: AAAAAGGAGGGGTGGATAAGAAGAGGAC 


\section{Genetic sex identification in yellow catfish}

Genomic DNA was respectively extracted from fin clips of the artificial breeding and wild groups yellow catfish. Then, the genomic DNA of each sample was used for PCR amplification to detect male/female genotype by specific primers designed from the genomic walking sequence. Further, the physical sex of yellow catfish was determined by anatomical dissection.

\section{Results}

\section{Genomic walking and sequence analysis of Y-linked marker Pf62-Y}

Fig.1A summarized the genomic walking process for Pf62-Y sequence. Firstly, the genomic DNA extracted from YY super-male was digested with 4 different restrict enzymes such as Dra I, Pvu II, EcoR V and $S t u \mathrm{I}$, and the digested fragments were ligated to adaptor respectively to construct four fragment pools. Then, the designed walking primers and adapter primers were used to amplify the corresponding genomic fragments from the constructed four DNA fragment pools, and the amplified fragments were sequenced. A total of 12 fragments were distinguished. As shown in Fig. 1A, three fragments (D1, D2 and D3) were amplified from the Dra I digested DNA fragment pool, two (P1 and P2) from the Pvu II pool, five (E1, E2, E3, E4 and E5) from EcoR V pool, and two (S1 and S2) from Stu I pool. The longest

A

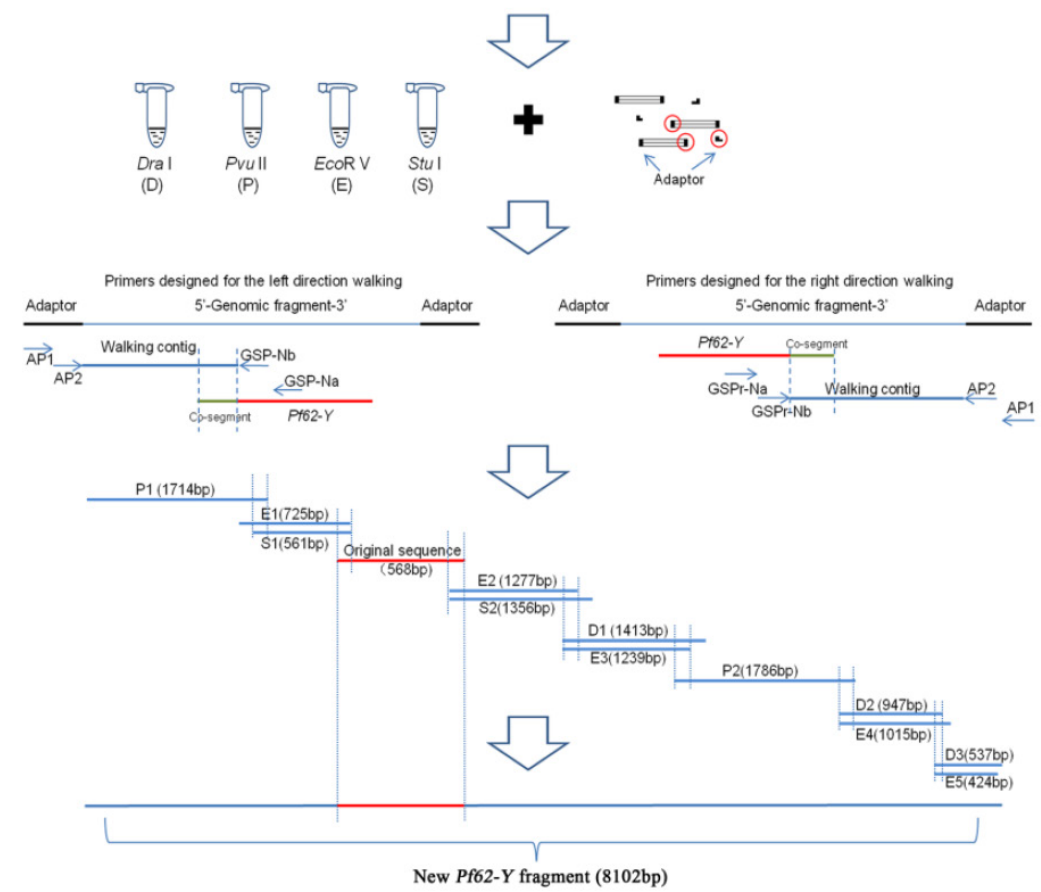

New Pf62-Y fragment (8102bp) fragment (P2) contained $1786 \mathrm{bp}$, and the shortest (E5) was only $424 \mathrm{bp}$. These walking fragment sequences and the original $P f 62-Y$ sequence were analyzed by DNAMAN software and assembled into a continuous 8102 bp Pf62-Y fragment (GenBank accession number: KF593811).

Moreover, an 8102 bp fragment was amplified from an YY super-male by PCR using L-A Taq polymerase (Fig.1B), and its sequence was identical to the assembled sequence from the walking fragments, which confirmed the genomic walking correction.

\section{Genetic differentiation between Pf62-Y and Pf62-X allele sequences}

To reveal genetic difference between $P f 62-Y$ and Pf62- $X$ alleles, we further obtained a 5362 bp Pf62-X allele sequence(GenBank accession number: KF593812) from a XX female individual by genomic walking and PCR amplification using degenerate primers. Sequence alignment of the 5362 bp Pf62-X allele and the corresponding $P f 62-Y$ allele shows that the nucleotide identity between them is $87.36 \%$, implicating that there exists substantial genetic differentiation between the Pf62-X and Pf62- $Y$ alleles (Fig.2). Interestingly, besides a large number of single nucleotide differences, Pf62- $Y$ allele has some small segment deletion in comparison with the corresponding $P f 62-X$ allele sequence. It seems to imply that the yellow catfish Y chromosome might be highly differentiated during the sex chromosome evolution process.
B

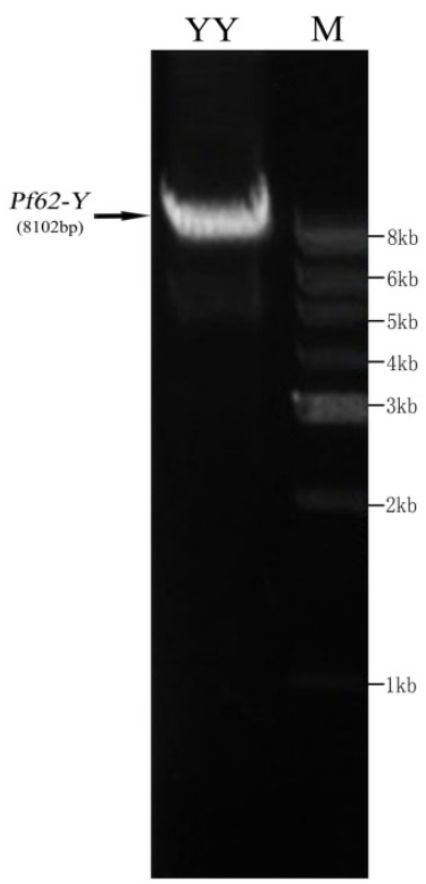

Fig I. Genomic walking process and sequence confirmation of $Y$-linked marker Pf62-Y. (A) The schematic diagram of genomic walking process for Pf62-Y sequence. (B) Detection of the 8102 bp Pf62-Y fragment amplified from an YY super-male in agarose gel. 


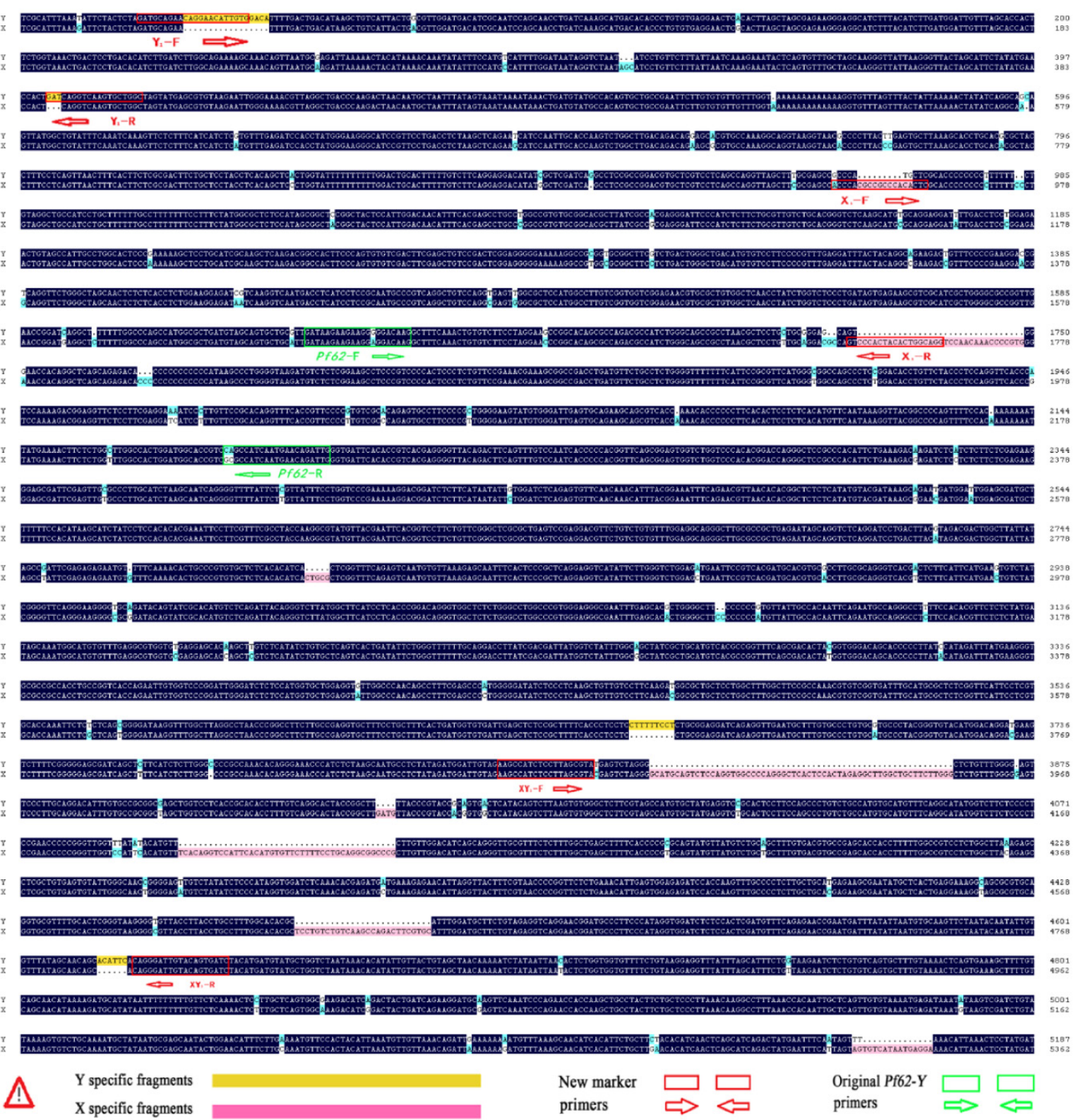

Fig 2. Sequence alignment between $P f 62-Y$ and $P f 62-X$ alleles. There are numerous single nucleotide differences and some small fragment deletion differences between $P f 62-Y$ and $P f 62-X$.

\section{Design of three pairs of new markers and ge- netic sex identification in $X X$ females, $X Y$ males and $Y Y$ super-males}

Based on the significant genetic difference between Pf62-Y and Pf62-X allele sequences, we further designed three pairs of new sequence-characterized primers, such as $Y$ chromosome-specific primer pair $Y_{1}-F$ and $Y_{1}-R, X$ chromosome-specific primer pair $X_{1}-F$ and $X_{1}-R$, as well as $Y$ and $X$ chromosomes-shared primer pair $X Y_{1}-F$ and $X Y_{1}-R$ (Fig. 2), to identify $Y Y$ super-males, $X Y$ males, and $X X$ females. As shown in Fig. 3, a $Y$ chromosome-specific 398 bp fragment is amplified by the primer pair of $Y_{1}-\mathrm{F}$ (GATGCAGAACAGGAACATTGTG) and $\mathrm{Y}_{1}-\mathrm{R}$
(GCCACCACTTGACCTGATC) from all the YY super-males and $X Y$ males, whereas no any product is observed from the $\mathrm{XX}$ females. An $\mathrm{X}$ chromosome-specific $823 \mathrm{bp}$ fragment is produced by the $\mathrm{X}_{1}-\mathrm{F}$ (ACCCACGCCGCCCACACTC) and $X_{1}-R$ (ACCTGCCAGTGTAGTGGGAC) primer pair from $X X$ females and $X Y$ males, while no any amplified sequence is obtained from the YY super-males. Owing to the existence of 4 fragment deletions on the Pf62-Y allele, two different size fragments (X-fragment: 955bp, Y-fragment: 826bp) are amplified by the primer pair of $\mathrm{XY}_{1}-\mathrm{F}$ (GATTGTAGAAGCCATCTC CTTAGCGTA) and $X Y_{1}-R$ (CATGTAGATCACTGT ACAATCCCTG) from the XY males, while only 955bp $\mathrm{X}$-fragment is observed in XX females, and only 826bp 
Y-fragment in $Y Y$ super-males. Therefore, three pairs of new genetic markers $\left(Y_{1}, X_{1}\right.$ and $\left.X Y_{1}\right)$ were developed to efficiently identify $Y Y$ super-males, $X Y$ males and $X X$ females, and the sex determination mechanism $\left(X_{+} \odot / X Y\right.$ ) $)$ of yellow catfish was further proofed by the $\mathrm{X}$ - and $\mathrm{Y}$-specific markers.

\section{Genetic sex identification of new markers in 5 different wild populations of yellow catfish}

In order to test detection efficiency and applicable scope of the three new genetic markers, we collected different wild population samples of yellow catfish from 5 segregate lakes, such as Liangzi Lake in Wuhan of Hubei, Chang Lake in Jingzhou of Hubei, Hong Lake in Honghu of Hubei, South Lake in Zhongxiang of Hubei, and Dongting Lake in Changde

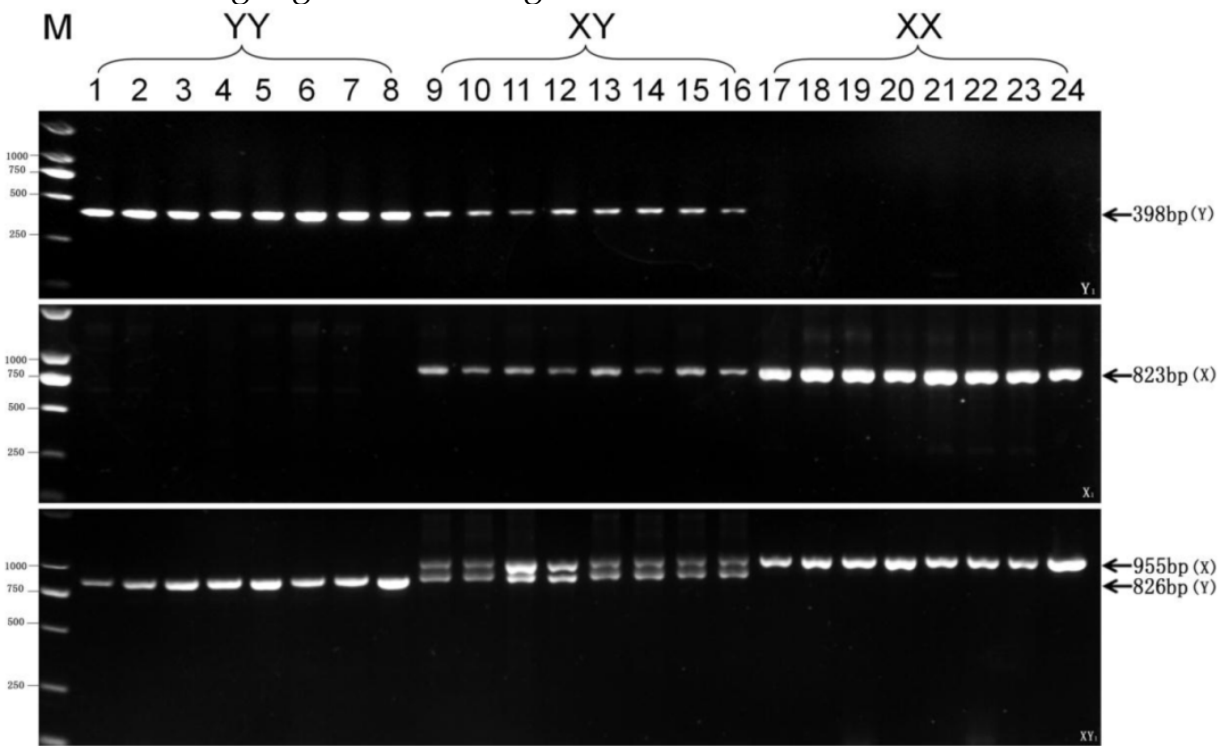

Fig 3. Test results of three pairs of new markers $\left(Y_{1}, X_{1}\right.$ and $\left.X Y_{1}\right)$ in $8 Y Y$ super-males, $8 X Y$ males, and $8 X X$ females respectively. The 398 bp $Y$-specific fragment amplified by $Y_{1}$ primer pair, the $823 \mathrm{bp} X$-specific fragment by $X_{1}$ primer pair, as well as 955 bp $X$-specific fragment and 826 bp $Y$-specific fragment by $X Y_{1}$, are indicated by arrows on the right. The DL2000 DNA marker sizes are shown on the left.

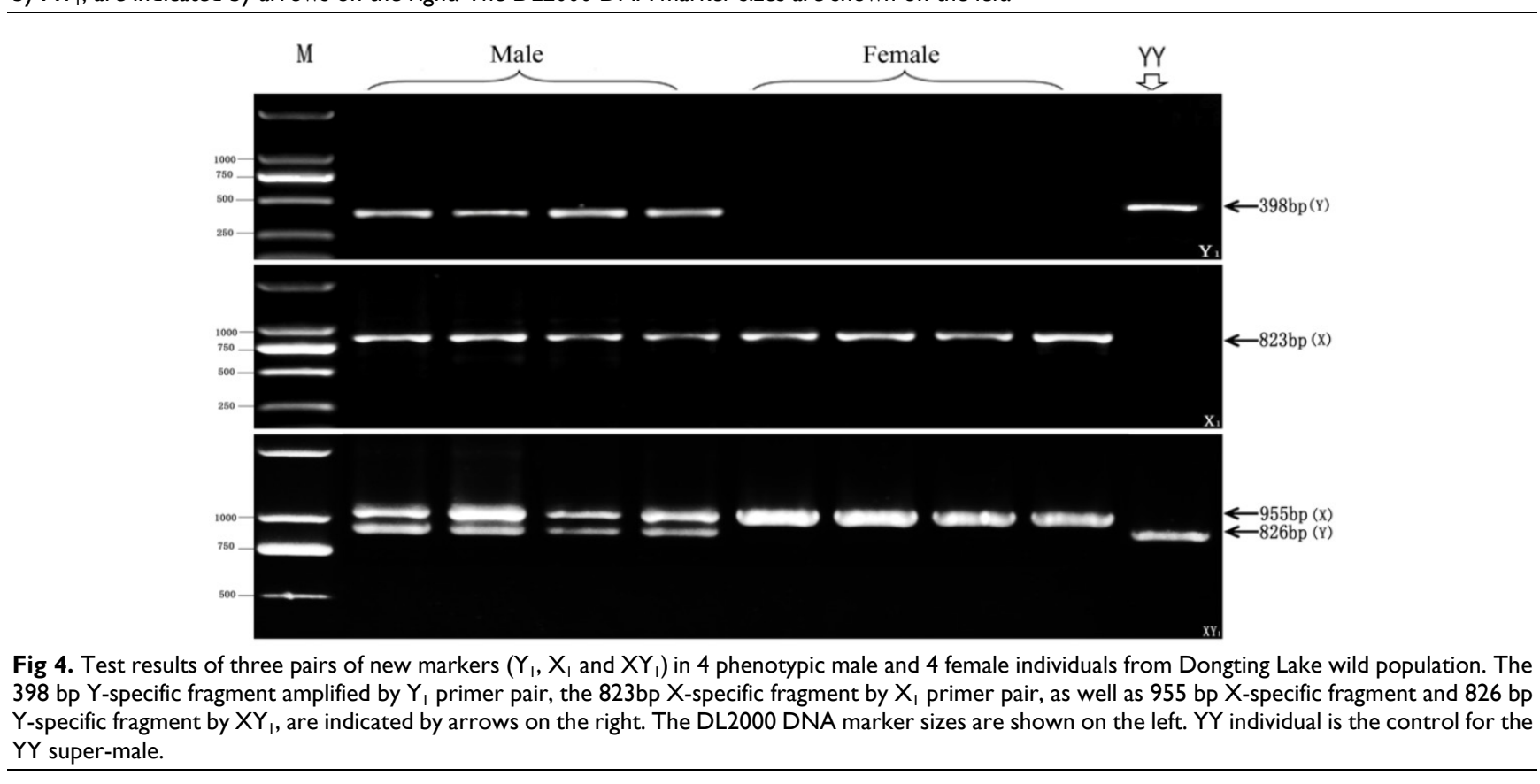

Fig 4. Test results of three pairs of new markers $\left(Y_{1}, X_{1}\right.$ and $\left.X Y_{1}\right)$ in 4 phenotypic male and 4 female individuals from Dongting Lake wild population. The 398 bp $Y$-specific fragment amplified by $Y_{1}$ primer pair, the 823 bp $X$-specific fragment by $X_{1}$ primer pair, as well as 955 bp $X$-specific fragment and 826 bp $Y$-specific fragment by $X Y_{1}$, are indicated by arrows on the right. The DL2000 DNA marker sizes are shown on the left. $Y Y$ individual is the control for the YY super-male.

of Hunan. The extracted genome DNAs were respectively used to identify genetic sex and their female or male phenotypes were affirmed by anatomizing ovary or testis. Fig. 4 shows the test results of three new genetic markers in Dongting Lake population samples. Consistent with the detection data in artificial breeding population, a Y chromosome-specific $398 \mathrm{bp}$ fragment is amplified by the primer pair of $\mathrm{Y}_{1}-\mathrm{F}$ and $\mathrm{Y}_{1}-\mathrm{R}$ only in male individuals, whereas an $X$ chromosome-specific $823 \mathrm{bp}$ fragment is detected by the $\mathrm{X}_{1}-\mathrm{F}$ and $\mathrm{X}_{1}-\mathrm{R}$ primer pair in both of females and males. And, two different size fragments (X-fragment: 955bp, Y-fragment: 826bp) are amplified by the primer pair of $X Y_{1}-F$ and $X Y_{1}-R$ from the $X Y$ males, while only $955 b p$ X-fragment is observed in $X X$ females. 


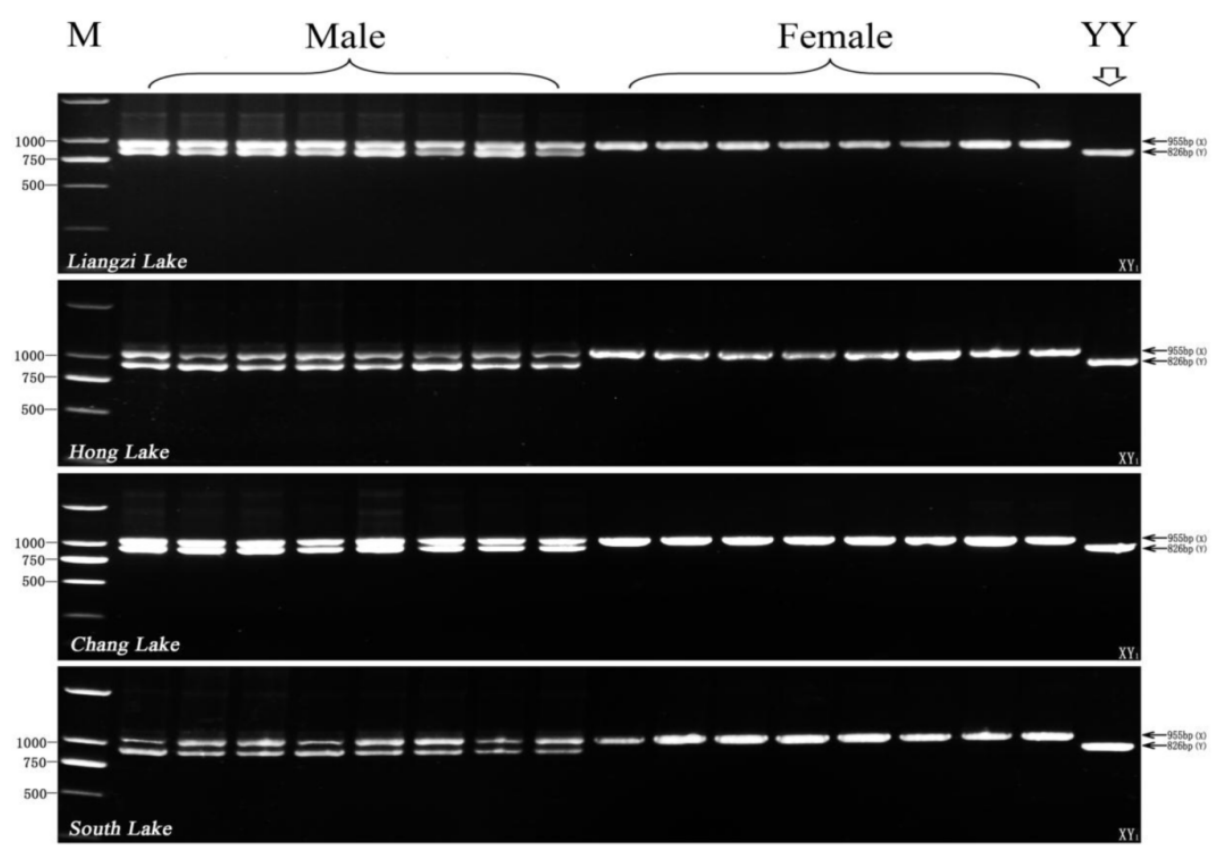

Fig 5. Test results of the $X Y$, marker in other four wild populations ( 8 male and 8 female individuals from each population) from Liangzi Lake (Wuhan), Hong Lake (Honghu) Chang Lake (Jingzhou), and South Lake (Zhongxiang). The 955 bp X-specific fragment and 826 bp Y-specific fragment amplified by XY, primer pair are indicated by arrows on the right. The DL2000 DNA marker sizes are shown on the left. YY individual is the control for the YY super-male.

Furthermore, we used $X Y_{1}-\mathrm{F}$ and $X Y_{1}-\mathrm{R}$ primer pair to test four other wild populations sampled from Liangzi Lake, Hong Lake, Chang Lake, and South Lake. As shown in Fig. 5, two different size fragments (X-fragment: 955bp, Y-fragment: 826bp) are amplified by the primer pair of $X Y_{1}-\mathrm{F}$ and $X Y_{1}-\mathrm{R}$ from all of the male individuals in 4 wild populations, and only 955bp X-fragment is detected from all of the four wild population female samples. Altogether, the three pairs of new genetic markers $\left(\mathrm{Y}_{1}, \mathrm{X}_{1}\right.$ and $\left.\mathrm{XY}_{1}\right)$ can efficiently use genetic sex identification of yellow catfish in all sampled populations, and their detection efficiency reaches to $100 \%$.

\section{Discussion}

In this study, we have cloned and sequenced the Y chromosome-linked marker Pf62-Y 8102 bp fragment by genome walking, and also obtained its corresponding 5362 bp Pf62-X allele sequence. Their corresponding sequence alignment reveal significant genetic difference between them, implicating that significant genetic differentiation has occurred between the corresponding $\mathrm{X}$ and $\mathrm{Y}$ chromosome regions. It should be a significant finding for this study. In theory, sex chromosome pair has been proposed to evolve from recombination suppression of a pair of autosomes, and the recombination suppression leads to further genetic differentiation and even hetromorphic sex chromosomes [34]. Recently, high degree of sex chromosome differentiation has been also revealed in stickleback fishes by using a set of mi- crosatellite markers [35].

Our previous study has demonstrated that the markers $P f 62-Y$ and $P f 62-X$ are respectively linked to $Y$ chromosome and $X$ chromosome, and they can be used to identify $X X$ females, $X Y$ males and $Y Y$ super-males [16], but the used SCAR primers have only two nucleotide difference, which restricts the wide utility because of nucleotide polymorphism. The genetic difference between $P f 62-Y$ and $P f 62-X$ allele sequences directly reflects genetic differentiation between $\mathrm{Y}$ chromosome and $\mathrm{X}$ chromosome. According to the significant genetic difference between $P f 62-Y$ and $P f 62-X$ allele sequences revealed by the current study, we have designed three pairs of sex-specific primers, and thereby developed three pairs of new genetic markers, such as $Y_{1}, X_{1}$ and $X Y_{1}$. It has been demonstrated that the three pairs of new genetic markers can efficiently identify $Y Y$ super-males, $X Y$ males and XX females in artificial breeding populations and can efficiently distinguish $X Y$ males and $X X$ females in different wild populations of yellow catfish. In summary, our molecular markers highlight a method for sustainable aquaculture of all-male yellow catfish in China.

\section{Acknowledgements}

This work was supported by grants from the special Fund for Agro-scientific Research in the Public Interest from the Ministry of Agriculture of China (2009030406), the National Key Basic Research Program (2010CB126301), the Innovation Project of Chi- 
nese Academy of Sciences (KSCX2-EW-N-04), and the Autonomous Project of State Key Laboratory of Freshwater Ecology and Biotechnology (2011FBZ17).

\section{Competing Interests}

The authors have declared that no competing interest exists.

\section{References}

1. Gui JF, Zhu ZY. Molecular basis and genetic improvement of economically important traits in aquaculture animals. Chin Sci Bull. 2012; 57: 1751-1760.

2. Zheng XH, Kuang YY, Lv WH, Cao DC, Zhang XF, Li C, Lu CY, Sun XW. A consensus linkage map of common carp (Cyprinus carpio L.) to compare the distribution and variation of QTLs associated with growth traits. Sci China Life Sci. 2013; 56: 351-359

3. Gui JF. Genetic basis and artificial control of sexuality and reproduction in fish. Beijing: Science Press. 2007.

4. Gui JF, Zhou L. Genetic basis and breeding application of clonal diversity and dual reproduction modes in polyploid Carassius auratus gibelio. Sci China Life Sci. 2010; 53: 409-415.

5. Piferrer F, Ribas L, Díaz N. Genomic approaches to study genetic and environmental influences on fish sex determination and differentiation. Mar Biotechnol. 2012; 14: 591-604.

6. Kovacs B, Egedi S, Bartfai R, Orban L. Male-specific DNA markers from African catfish (Clarias gariepinus). Genetica. 2000; 110: 267-276.

7. Ezaz MT, Harvey SC, Boonphakdee C, Teale AJ, McAndrew BJ, Penman DJ. Isolation and physical mapping of sex-linked AFLP markers in nile tilapia (Oreochromis niloticus L.). Mar Biotechnol. 2004; 6: 435-445.

8. Lee BY, Penman DJ, Kocher TD. Identification of a sex-determining region in Nile tilapia (Oreochromis niloticus) using bulked segregant analysis. Anim Genet. 2003; 34: 379-383.

9. Lee BY, Coutanceau JP, Ozouf-Costaz C, D'Cotta H, Baroiller JF, Kocher TD. Genetic and physical mapping of sex-linked AFLP markers in Nile tilapia (Oreochromis niloticus). Mar Biotechnol. 2011; 13: 557-562.

10. Liu F, Sun F, Li J, Xia JH, Lin G, Tu RJ, Yue GH. A microsatellite-based linkage map of salt tolerant tilapia (Oreochromis mossambicus x Oreochromis spp.) and mapping of sex-determining loci. BMC Genomics 2013;14: 58.

11. Yue GH, Ong D, Wong CC, Lim LC, Orban L. A strain-specific and a sex-associated STS marker for Asian arowana (Scleropages formosus, Osteoglossidae). Aqua Res. 2003; 34:951-957.

12. Felip A, Young WP, Wheeler PA, Thorgaard GH. An AFLP-based approach for the identification of sex-linked markers in rainbow trout (Oncorhynchus mykiss). Aquaculture. 2005; 247: 35-43.

13. Chen SL, Li J, Deng SP, Tian YS, Wang QY, Zhuang ZM, Sha ZX, Xu JY. Isolation of female-specific AFLP markers and molecular identification of genetic sex in half-smooth tongue sole (Cynoglossus semilaevis). Mar Biotechnol. 2007; 9: 273-280.

14. Chen SL, Deng SP, Ma HY, Tian YS, Xu JY, Yang JF, Wang QY, Ji XS, Shao CW, Wang XL, Wu PF, Deng H, Zhai JM. Molecular marker-assisted sex control in half-smooth tongue sole (Cynoglossus semilaevis). Aquaculture. 2008; 283: 7-12.

15. Ma Q, Liu S, Zhuang Z, Lin L, Sun Z, Liu C, Ma H, Su Y, Tang Q. Genomic structure, polymorphism and expression analysis of the growth hormone (GH) gene in female and male Half-smooth tongue sole (Cynoglossus semilaevis). Gene. 2012; 493: 92-104.

16. Wang D, Mao HL, Chen HX, Liu HQ, Gui JF. Isolation of Y- and X-linked SCAR markers in yellow catfish and application in the production of all-male populations. Anim Genet. 2009; 40: 978-981.

17. Chen J, Wang Y, Yue Y, Xia X, Du Q, Chang Z. A novel male-specific DNA sequence in the common carp, Cyprinus carpio. Mol Cell Probes. 2009; 23: 235-239.

18. Chen JJ, Du QY, Yue YY, Dang BJ, Chang ZJ. Screening and identification of male-specific DNA fragments in common carps Cyprinus carpio using suppression subtractive hybridization. J Fish Biol. 2010; 77: 403-413.

19. Xia XH, Zhao J, Du QY, Zhi JH, Chang ZJ. Cloning and identification of a female-specific DNA marker in Paramisgurnus dabryanus. Fish Physiol Biochem. 2011; 37: 53-59.

20. da Silva EM, Wong MS, Martins C, Wasko AP. Screening and characterization of sex-specific DNA fragments in the freshwater fish matrincha, Brycon amazonicus (Teleostei: Characiformes: Characidae). Fish Physiol Biochem. 2012; 38: 1487-96.

21. Viñas A, Taboada X, Vale L, Robledo D, Hermida M, Vera M, Martínez P. Mapping of DNA sex-specific markers and genes related to sex differentiation in turbot (Scophthalmus maximus). Mar Biotechnol. 2012; 14: 655-663.

22. Rao HO, Deng JC, Wang WM, Gao ZX. An AFLP-based approach for the identification of sex-linked markers in blunt snout bream, Megalobrama amblycephala (Cyprinidae). Genet Mol Res. 2012; 11: 1027-1031.

23. $\mathrm{Xu} \mathrm{D}$, Lou $\mathrm{B}, \mathrm{Xu} \mathrm{H}, \mathrm{Li} \mathrm{S}$, Geng $\mathrm{Z}$. Isolation and characterization of male-specific DNA markers in the rock bream Oplegnathus fasciatus. Mar Biotechnol. 2013; 15: 221-229.
24. Liu HQ, Cui SQ, Hou CC, Xu J, Chen HX. YY super-male generated gynogenetically from XY female in Pelteobagrus fulvidraco (Richardson). Acta Hydrobiologica Sinica. 2007; 31: 718-725.

25. Liu HQ, Guan B, Xu J, Hou CC, Tian H, Chen HX. Genetic manipulation of sex ratio for the large-scale breeding of $Y Y$ super-male and $X Y$ all-male yellow catfish (Pelteobagrus fulvidraco Richardson). Mar Biotechnol. 2013; 15: 321-328

26. Zhong L, Chen J, Cai Y, Pan J, Xia A. Research progress in DNA molecular markers of yellow catfish Pseudobagrus fulvidraco. Chinese Agriculture Science Bulletin.2012; 28: 67-75

27. Sambrook J, Fritsch EF, Maniatis T. Molecular cloning: A laboratory manual, 2nd ed. Cold Spring Harbor, NY: Cold Spring Harbor Laboratory Press. 1989: 463-468.

28. Zhou L, Wang Y, Gui JF. Genetic evidence for gonochoristic reproduction in gynogenetic silver crucian carp (Carassius auratus gibelio bloch) as revealed by RAPD assays. J Mol Evol. 2000; 51: 498-50

29. Xia W, Zhou L, Yao B, Li CJ, Gui JF. Differential and spermatogenic cell-specific expression of DMRT1 during sex reversal in protogynous hermaphroditic groupers. Mol Cell Endocrinol. 2007; 263: 156-172.

30. Huang W, Zhou L, Li Z, Gui JF. Expression pattern, cellular localization and promoter activity analysis of ovarian aromatase (Cyp19a1a) in protogynous hermaphrodite red-spotted grouper. Mol Cell Endocrinol, 2009; 307: 224-236.

31. Li FB, Gui JF. Clonal diversity and genealogical relationships of gibel carp in four hatcheries. Anim Genet. 2008; 39: 28-33.

32. Xu S, Xia W, Zohar Y, Gui JF. Zebrafish dmrta2 regulates the expression of $c d k n 2 c$ in spermatogenesis in the adult testis. Biol. Reprod. 2013; 88(14): 1-12

33. Jiang FF, Wang ZW, Zhou L, Jiang L, Zhang XJ, Apalikova OV, Brykov VA, Gui JF. High male incidence and evolutionary implications of triploid form in northeast Asia Carassius auratus complex. Mol Phylogenet Evol. 2013; 66: 350-359.

34. Charlesworth D, Charlesworth B, Marais G. Steps in the evolution of heteromorphic sex chromosomes. Heredity. 2005; 95: 118-128.

35. Shikano T, Natri HM, Shimada Y, Merila J. High degree of sex chromosome differentiation in stickleback fishes. BMC Genomics. 2011; 12: 474. 